\title{
Initiation and evolution of phase separation in heteroepitaxial InAIAs films
}

\author{
B. Shin, A. Lin, K. Lappo, and R. S. Goldman ${ }^{\text {a) }}$ \\ Department of Materials Science and Engineering, University of Michigan, Ann Arbor, \\ Michigan 48109-2136 \\ M. C. Hanna, S. Francoeur, A. G. Norman, and A. Mascarenhas \\ National Renewable Energy Laboratory, Golden, Colorado 80401
}

(Received 7 January 2002; accepted for publication 5 March 2002)

\begin{abstract}
We have investigated the initiation and evolution of phase separation in heteroepitaxial InAlAs films. In misfit-free InAlAs layers, cross-sectional scanning tunneling microscopy (XSTM) reveals the presence of isotropic nanometer-sized clusters. For lattice-mismatched InAlAs layers with $1.2 \%$ misfit, quasiperiodic contrast modulations perpendicular to the growth direction are apparent. Interestingly, these lateral modulations are apparently initiated within the first few bilayers of film growth, and both the amplitude and wavelength of the modulations increase with film thickness. The saturation value of the modulation wavelength determined from XSTM coincides with the lateral superlattice period determined from (002) x-ray reciprocal space maps, suggesting that the lateral modulation wavelength represents a periodic composition variation. Together, these results suggest that phase separation in the heteroepitaxial InAlAs thin-film system is a misfit-driven kinetic process initiated by random compositional nonuniformities, which later develop into coupled compositional and surface morphological variations. (C) 2002 American Institute of Physics.
\end{abstract}

[DOI: $10.1063 / 1.1476386]$

In recent years, compound semiconductor alloys have been incorporated into a wide range of electronic and optoelectronic devices. In most of these systems, growth conditions have been reported for which phase separation occurs. Yet, the thermodynamic versus the kinetic origin of phase separation, as well as the experimental conditions for determining the presence of phase separation have been the subject of debate for nearly 20 years. ${ }^{1,2}$ Furthermore, in latticemismatched thin-film systems, the relative roles of morphological undulations and random compositional nonuniformities in the initiation of phase separation remain controversial. For example, in the InAlAs system, $\langle 110\rangle$-oriented phase separation has been observed in bulk-like epilayers ${ }^{3-6}$ and in short period superlattices ${ }^{7}$ grown well above the miscibility gap temperature. In those cases, phase separation was attributed to spinodal decomposition, ${ }^{4}$ morphological undulations, ${ }^{6,7}$ and differences in $\mathrm{Al}$ and In sticking coefficients on stepped surfaces. ${ }^{5}$ In this letter, we report nanometer-scale investigations of the initiation and evolution of $\langle 110\rangle$-oriented phase separation in the heteroepitaxial InAlAs thin film system. Our combined cross-sectional scanning tunneling microscopy (XSTM) and x-ray reciprocal space map (XRSM) studies suggest that phase separation is a misfit-driven kinetic process initiated by random compositional nonuniformities, which later develop into coupled compositional variations and morphological undulations. This mechanism is likely to be applicable to a wide range of lattice-mismatched thin-film systems.

The samples were grown on (001)-oriented $p^{+}$InP substrates, using metalorganic chemical vapor deposition at a pressure of 100 mbar, with trimethylindium, trimethylaluminum, $\mathrm{AsH}_{3}$, and $\mathrm{PH}_{3}$ as source gases. The targeted structures included ten periods of $\mathrm{Zn}$-doped InAlAs/InP superlattices

a)Electronic mail: rsgold@engin.umich.edu consisting of alternating $20 \mathrm{~nm} \mathrm{InP}$ and $20 \mathrm{~nm}$ InAlAs grown at $620^{\circ} \mathrm{C}$, followed by $500 \mathrm{~nm}$ of undoped InAlAs grown at $500^{\circ} \mathrm{C}$. The entire structure was capped with $200 \mathrm{~nm} \mathrm{InP}$ grown at $620^{\circ} \mathrm{C}$. We will refer to the InAlAs/InP superlattices (SLS) as "vertical SL" and the $500 \mathrm{~nm}$ InAlAs layers as "bulk-like InAlAs."

High-resolution x-ray rocking curves (XRC) and XRSMs were measured with a Bede D1 x-ray diffractometer using $\mathrm{Cu} K \alpha$ radiation. Analysis of a series of (004) and (224) XRCs indicates that the vertical SL's are lattice matched to the InP substrate while the bulk-like InAlAs has an average In concentration of $71 \%$, and a misfit of $1.2 \%$ with respect to the InP substrate. For XSTM, the samples were cleaved to expose a (110) surface, in an ultrahigh vacuum (UHV) chamber with base pressure $<5 \times 10^{-11}$ Torr. ${ }^{8}$ Scanning tunneling microscopy was performed with commercially available Pt/Ir tips. All images were obtained with a constant tunnel current and sample bias voltages specified below.

An XRSM collected near the InP (002) reflection, with the $Q_{z}$ and $Q_{x}$ axes aligned parallel to the [001] and [110] directions, is shown in Fig. 1. Near the center of the map, the InP (002) and surrounding satellite peaks of the vertical SL are apparent. Left of the InP substrate peak, a peak corresponding to the bulk-like InAlAs is surrounded by first-order satellites of a lateral SL. Using the spacing between the peak associated with the bulk-like InAlAs and the first-order satellite peaks of the lateral SL, $\Delta Q_{x}$, we find a lateral SL period, $2 \pi / \Delta Q_{x},{ }^{9}$ equal to $17 \pm 1 \mathrm{~nm}$. Since these satellite peaks are present in (002) but not in (004) XRSM's, the lateral SL is primarily due to chemical or compositional variations, presumably resulting from alloy phase separation occurring at the surface during growth.

Both the vertical SL and the bulk-like InAlAs are evident in the large-scale XSTM image in Fig. 2(a). The InAlAs 


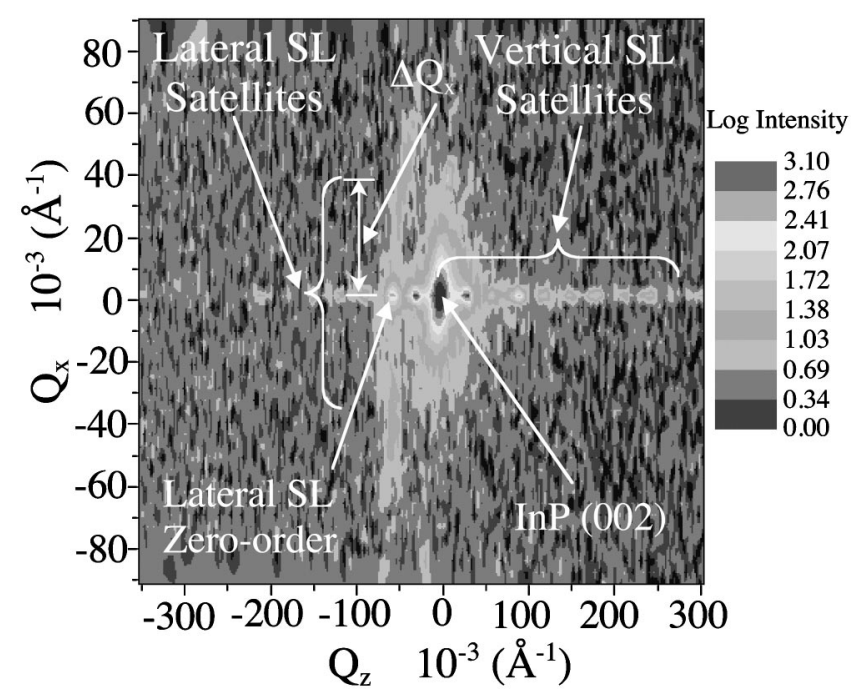

FIG. 1. X-ray reciprocal space map of InAlAs/InP heterostructure collected near the InP (002) reflection.

layers within the vertical SL contain isotropic clusters with typical diameters of $\sim 2 \mathrm{~nm}$ and separations ranging from 5 to $10 \mathrm{~nm}$, similar to an earlier report. ${ }^{10}$ The bulk-like InAlAs contains quasiperiodic contrast modulations perpendicular to the growth direction. Since the lateral contrast modulations are present in the lattice-mismatched bulk-like InAlAs, but not in the misfit-free InAlAs layers within the latticematched vertical SL, their formation is likely related to the relaxation of misfit strain.

To investigate the initiation and evolution of the lateral contrast modulations, line cuts from Fig. 2(a) were collected at various distances from the vertical SL/bulk-like InAlAs interface, as shown in Fig. 2(b). The tip height variations in the line cuts, which correspond to contrast modulations in the XSTM images, are due to a combination of lateral chemical variations resulting from alloy phase separation, and surface distortions resulting from elastic relaxation of strain occurring during cleaving. ${ }^{11}$ Average modulation spacings, or apparent contrast modulation wavelengths, $\lambda$, were determined from positions throughout the thickness of the bulk-

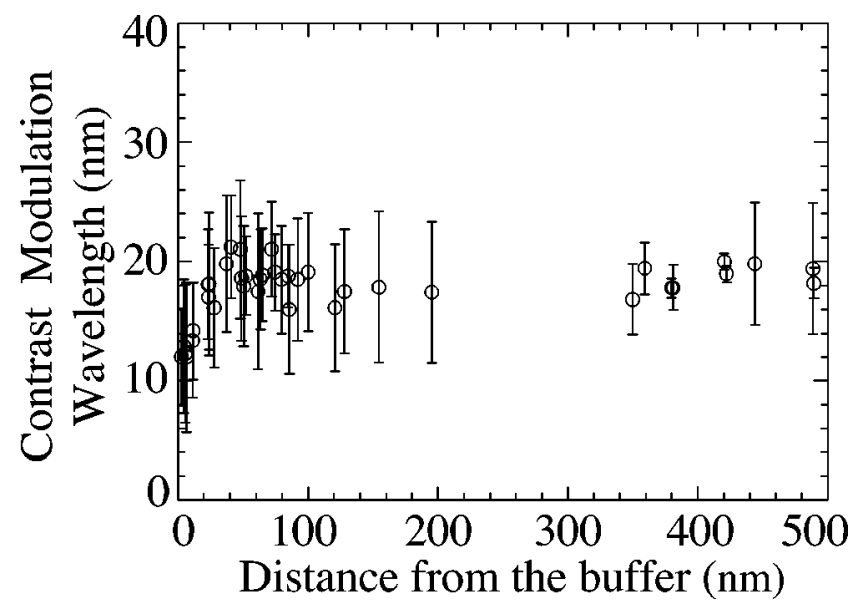

FIG. 3. Plot of contrast modulation wavelength vs distance from the interface between the vertical SL and the bulk-like InAlAs.

like InAlAs. Figure 3 shows $\lambda$ values from several largescale XSTM images, plotted as a function of distance from the interface between the vertical SL and the bulk-like InAlAs. We note that in determining $\lambda$ values, only those features in line cuts such as in Fig. 2(b), with heights at least twice the typical noise level $(\sim 0.4 \AA)$ were considered. It is evident that $\lambda$ increases with film thickness, reaching a saturation value of $\sim 20 \mathrm{~nm}$ at a film thickness $\sim 25 \mathrm{~nm}$. This saturation value of $\lambda$ is within $13 \%$ of the lateral SL period determined from the (002) XRSM, suggesting that $\lambda$ in XSTM images corresponds to the wavelength of lateral composition variations.

Interestingly, the quasiperiodic lateral contrast modulations are observed in the line cuts within only a few bilayers of the vertical SL/bulk-like InAlAs interface. Furthermore, the average modulation spacings increase with film thickness, due mainly to the merging of modulations, an example of which is indicated by vertical arrows in Fig. 2(b). As the modulations merge, the modulation amplitude increases, due mainly to an increase in elastic strain relaxation occurring during cleaving.

An XSTM image in the vicinity of the interface between

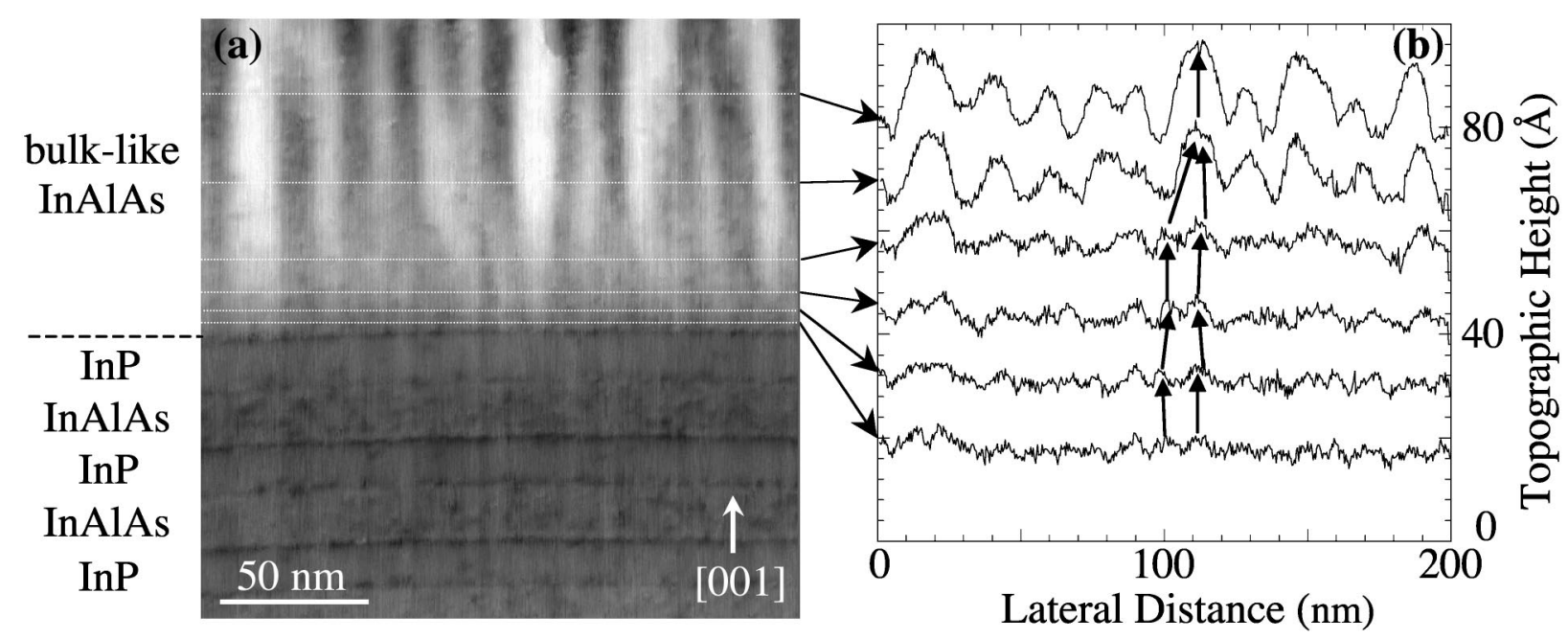

FIG. 2. Large-scale (a) XSTM topographic image showing both InAlAs/InP vertical SLs and bulk-like InAlAs, exhibiting lateral superlattices. The image was acquired at a sample bias voltage of $-2 \mathrm{~V}$, and the gray-scale range displayed is (a) $20 \AA$. Cuts of the tip height along various lines in (a) are plotted in (b). The merging of a pair of peaks is indicated by vertical arrows in (b). 




FIG. 4. Large-scale XSTM topographic image at the interface between the InP cap layer and the bulk-like InAlAs, acquired at sample bias voltage of $-1.8 \mathrm{~V}$. The gray-scale range displayed is $24 \AA$.

the bulk-like InAlAs and the InP cap is shown in Fig. 4. In the lower region of Fig. 4, lateral contrast modulations within the bulk-like InAlAs are apparent. The lateral contrast modulations persist up to a cleavage-induced surface step, whose shape resembles a morphological undulation. If the position of the cleavage-induced surface step coincides with the interface between the bulk-like InAlAs and the InP cap, the growth front has developed a morphological undulation, with crests and troughs correlated with the lateral contrast modulations.

Modulation wavelengths of the same order of magnitude have been reported for similar films with significantly smaller misfits. ${ }^{3}$ For phase separation initiated by morphological undulations, classical instability theory predicts an undulation wavelength inversely proportional to the misfit squared. ${ }^{12,13}$ However, the fact that this proportionality is not observed experimentally suggests that alternative models need to be considered. Therefore, we propose a model in which phase separation is initiated by random compositional nonuniformities occurring during the early stages of film growth. As more layers are deposited, the phase-separated clusters merge together to more effectively relax misfit strain. Consequently, the spacing between phase-separated regions increases until it is presumably limited by surface diffusion. Meanwhile, the merging of domains also leads to the development of surface undulations which further drive the phase-separation process. Interestingly, Spencer et al. ${ }^{14}$ recently proposed that under conditions of differing mobilities of alloying species, such as in our case where InAs is predicted to have 50 times higher surface diffusivity than AlAs ${ }^{15}$ compositional variations may develop in the absence of surface undulations.

In summary, we investigated the initiation and evolution of phase separation in heteroepitaxial InAlAs films using
UHV-XSTM and XRSM. In misfit-free InAlAs layers, isotropic nanometer-sized clusters are apparent. For 1.2\% mismatched InAlAs films grown at lower temperature, XSTM reveals quasiperiodic contrast modulations perpendicular to the growth direction. The lateral modulations are apparently initiated in the initial stages of film growth, and the modulation wavelength increases with film thickness. The saturation modulation wavelength is consistent with the XRSM lateral periodicity, suggesting that the wavelength of the XSTM contrast modulations represents a periodic compositional variation. These results suggest that misfit is the driving force for the observed phase separation. We note that the initiation and evolution of phase separation may also be affected by the growth temperature. ${ }^{16}$ In any case, it is likely that phase separation in the heteroepitaxial InAlAs thin film system is initiated by random compositional nonuniformities, which later develop into coupled compositional and surface morphological variations.

The authors acknowledge S. R. Lee for useful discussions. This work was supported in part by the National Science Foundation (Grant No. 9773707), the donors of the Petroleum Research Fund, administered by the American Chemical Society, and the DoD Multidisciplinary University Research Initiative (MURI) administered by the Army Research Office under Grant No. DAAD 19-01-1-0462. One of the authors (K.L.) was supported by a Marian Sarah Parker Scholarship.

${ }^{1}$ G. B. Stringfellow, J. Cryst. Growth 65, 454 (1983).

${ }^{2}$ A. Zunger and S. Mahajan, Handbook on Semiconductors (NorthHolland, Amsterdam, 1994), Vol. 3, p. 1399.

${ }^{3}$ H. K. Cho, J. Y. Lee, M. S. Kwon, B. Lee, J. H. Baek, and W. S. Han, Mater. Sci. Eng., B 64, 174 (1999).

${ }^{4}$ S. W. Jun, T.-Y. Seong, J. H. Lee, and B. Lee, Appl. Phys. Lett. 68, 3443 (1996).

${ }^{5}$ F. Peiró, A. Cornet, J. C. Ferrer, J. R. Morante, G. Halkias, and A. Georgakilas, Mater. Res. Soc. Symp. Proc. 419, 265 (1996).

${ }^{6}$ G. Grenet, M. Gendry, M. Oustric, Y. Robach, L. Porte, G. Hollinger, O. Marty, M. Pitaval, and C. Priester, Appl. Surf. Sci. 123, 324 (1998).

${ }^{7}$ J. M. Millunchick, R. D. Twesten, D. M. Follstaedt, S. R. Lee, E. D. Jones, Y. Zhang, S. P. Ahrenkiel, and A. Mascarenhas, Appl. Phys. Lett. 70, 1402 (1997).

${ }^{8}$ B. Lita, R. S. Goldman, J. D. Phillips, and P. K. Bhattacharya, Appl. Phys. Lett. 74, 2824 (1999).

${ }^{9}$ S. R. Lee, J. Mirecki Millunchick, R. D. Twesten, D. M. Follstaedt, J. L. Reno, S. P. Ahrenkiel, and A. G. Norman, J. Mater. Sci. 10, 191 (1999).

${ }^{10}$ M. L. Seaford, W. Wu, K. G. Eyink, D. H. Tomich, J. R. Tucker, and L. F. Eastman, J. Vac. Sci. Technol. B 15, 1274 (1997).

${ }^{11}$ R. S. Goldman, R. M. Feenstra, C. Silfvenius, B. Stålnacke, and G. Landgren, J. Vac. Sci. Technol. B 15, 1027 (1997).

${ }^{12}$ R. J. Asaro and W. A. Tiller, Metall. Trans. 3, 1789 (1972).

${ }^{13}$ D. J. Srolovitz, Acta Metall. 37, 621 (1989).

${ }^{14}$ B. J. Spencer, P. W. Voorhees, and J. Tersoff, Appl. Phys. Lett. 76, 3022 (2000).

${ }^{15}$ T. Shitara, D. D. Vvedensky, J. H. Neave, and B. A. Joyce, Mater. Res. Soc. Symp. Proc. 312, 267 (1993).

${ }^{16}$ S. Francoeur, M. C. Hanna, A. G. Norman, and A. Mascarenhas, Appl. Phys. Lett. 80, 243 (2002). 